Research Paper

\title{
A Novel Nomogram for Predicting the Survival of Patients with Invasive Upper Tract Urothelial Carcinoma
}

\author{
Zaishang $\mathrm{Li}^{1,2,3 \#}$, Xueying $\mathrm{Li}^{4 \#}$, Yonghong Li ${ }^{5,6,7 \#}$, Ying $\mathrm{Liu}^{1,2,3}$, Peng $\mathrm{Du}^{8}$, Zenqing $\mathrm{Liu}^{1,2,3 凶}$ and Kefeng Xiao ${ }^{1,2,3}$ \\ 1. Department of Urology, Shenzhen People's Hospital, The Second Clinic Medical College of Jinan University 518060, Shenzhen, Guangdong, P. R. China. \\ 2. Department of Urology, First Affiliated Hospital of Southern University of Science and Technology, 518060, Shenzhen, Guangdong, P. R. China. \\ 3. Department of Urology, Shenzhen Engineering and Technology Center of minimally Invasive Urology, Shenzhen People's Hospital, 518060, Shenzhen, Guangdong, P. \\ R. China. \\ 4. Department of Oncology, The Seventh Affiliated Hospital Sun Yat-sen University, 518107, Shenzhen, Guangdong, P. R. China. \\ 5. Department of Urology, Sun Yat-sen University Cancer Center, 510060, Guangzhou, Guangdong, P. R. China. \\ 6. State Key Laboratory of Oncology in South China, 510060, Guangzhou, Guangdong, P. R. China. \\ 7. Collaborative Innovation Center of Cancer Medicine, 510060, Guangzhou, Guangdong, P. R. China. \\ 8. Gynecology Department, Long-gang District Maternal and Child Healthcare Hospital, 518172, Shenzhen, Guangdong, P. R. China. \\ \#These authors contributed equally to this research. \\ $\triangle$ Corresponding authors: Professor Ke-Feng Xiao and Dr. Zengqing Liu, Address: Department of Urology, Shenzhen People's Hospital, 518060, Shenzhen, Guangdong, P. \\ R. China. E-mail: kevin5510315@qq.com; evans9902@163.com.
}

(C) The author(s). This is an open access article distributed under the terms of the Creative Commons Attribution License (https://creativecommons.org/licenses/by/4.0/). See http://ivyspring.com/terms for full terms and conditions.

Received: 2020.07.07; Accepted: 2020.10.30; Published: 2021.01.01

\begin{abstract}
Purpose: Available tools for the prediction of the prognosis of patients with upper tract urothelial carcinoma (UTUC) are unified. We determined whether a novel nomogram is effective in estimating the survival of patients with invasive UTUC.

Methods: From January 2004 to December 2015, 4796 invasive UTUC patients in the Surveillance, Epidemiology and End Results database underwent radical nephroureterectomy (RNU) for invasive UTUC. The medical records of the patients were randomly (7:3) divided into the training and validation cohorts. The independent factors included in the nomogram were selected by multivariate analyses. The nomogram was developed based on the training cohort. Bootstrap validation was applied to validate the nomogram, whereas external validation was performed using the validation cohort. The accuracy and discrimination of the nomogram were assessed using concordance indices ( $\mathrm{C}$-indices) and calibration curves.

Results: The multivariate Cox regression model identified that age, tumor stage, node stage, metastasis stage and grade were associated with survival. In the training set, the nomogram, which included the above factors, exhibited discrimination power superior to that of the 8th American Joint Committee on Cancer (AJCC) TNM classification (Harrell's C-index, 0.74 vs. $0.71 ; P<0.001$ ). The nomogram showed better probability of survival agreement with the C-index than the AJCC-TNM staging system in the bootstrap validation ( 0.74 vs. 0.70 ; $P<0.001$ ) and validation set (Harrell's $C$-index, 0.77 vs. $0.73 ; P<0.001$ ). The validation revealed that this nomogram exhibited excellent discrimination and calibration capacities.

Conclusion: An accurate novel nomogram that is superior to the current AJCC-TNM staging system was established for the prediction of CSS after RNU for invasive UTUC.
\end{abstract}

Key words: urothelial carcinoma, upper urinary tract, prognosis, radical nephroureterectomy, mortality

\section{Introduction}

Urothelial carcinomas (UCs) are the fourth most common tumors. However, upper tract urothelial carcinomas (UTUCs) are uncommon and account for only $5-10 \%$ of all UCs, and $60 \%$ of UTUCs are invasive at diagnosis [1, 2]. Radical nephroureterectomy (RNU) is the standard surgical treatment for patients with invasive UTUC $[3,4]$.
Due to individual differences, patients' prognosis significantly varies. Patients' prognosis can be predicted by clinicopathological factors, such as the $\mathrm{T}$ stage, $\mathrm{N}$ stage and grade [5-7]. However, the prognosis of patients cannot equally and accurately be reflected by a single factor. The European Association of Urology Guidelines on Upper Urinary Tract 
Urothelial Carcinoma refers to several nomograms [8]. Different nomograms incorporating various variables have been developed and are even based on the same French collaborative national database [9-11]. Several nomograms include blood parameters, radiographic parameters or immunohistochemical markers [12, 13]. Other nomograms include $\geq 6$ clinicopathological features [10, 14]. Therefore, a unified standard for nomograms is lacking.

The purpose of this study is to explore whether common factors could be used to develop a concise prediction model. To address the void in the ability to predict the prognosis of patients with UTUC, we developed a new model for the prediction of cancerspecific mortality (CSS) after RNU.

\section{Materials and methods}

\section{Study population}

Patients diagnosed with UTUC (codes ICD-O-2 C65.9 and C66.9) between 2004 and 2015 with available TNM stage classification data were identified from 18 Surveillance, Epidemiology and End Results (SEER) registries. Data from Alaska; Atlanta; California, except for San Francisco (SF)/San Jose/Monterey (SJM)/Los Angeles (LA); Connecticut; Detroit; Greater Georgia; Hawaii; Kentucky; Los Angeles; Rural Georgia; Louisiana; Iowa; New Jersey; New Mexico; San Francisco; San Jose; Seattle; and Utah were obtained. An independent pathological committee reviewed all histopathology findings [15].

Patients with detailed clinicopathological information who underwent RNU for invasive UTUC were considered. The detailed eligibility criteria were as follows: 1) histologically confirmed UTUC, 2) RNU for a primary lesion, 3) definite pathological staging, 4) detailed medical records, and 5) follow-up $\geq 1$ month. The data with the highest level of histopathology or data concerning the primary tumor were considered.

\section{Methods}

The cause of death was defined according to the SEER mortality cause assignment. The outcome of interest was CSS. The medical records of the patients were randomly (7:3) divided into the training $(n=3360)$ and validation $(n=1436)$ cohorts. The Kaplan-Meier method was used to determine CSS.

The log-rank test was used to compare the CSS rates among the different groups. A logistical Cox regression model was used to select the most useful prognostic markers of CSS. For the AJCC-pathological prognostic group classification, including the tumor stage, node stage, and metastasis stage, only the latter factors were included in the multivariate analysis. Accordingly, a nomogram for the prediction of the individual probability of CSS was developed based on the training cohort. Bootstrap validation (resampling with 1000 iterations) was applied to validate the nomogram, whereas external validation was performed using the validation cohort.

For the model validation, we assessed its discrimination and calibration. Discrimination was measured using the c-index. The statistical analyses were performed, and a two-sided $\mathrm{P}<0.05$ was considered significant. The models, statistical analysis, and figures were prepared using SAS 9.4 software version (Cary, NC) and R 3.5.1 (http://www.cran.r-project.org).

\section{Results}

In total, 4796 patients who underwent RNU for invasive UTUC were randomly (7:3) divided into the training $(n=3360)$ and validation $(n=1436)$ cohorts. In the training cohort, the median age at diagnosis was 73 years (range, 30-96 years). The median age at diagnosis in the validation cohort was 73 years (range, 38-101 years). Table 1 shows the detailed clinicopathological characteristics of the cohorts.

Table 1. Clinicopathological characteristics of the UTUC patients

\begin{tabular}{|c|c|c|c|}
\hline Variable & $\begin{array}{l}\text { Training set } \\
(\mathrm{n}=3360)\end{array}$ & $\begin{array}{l}\text { Validation set } \\
(\mathrm{n}=1436)\end{array}$ & $P$ \\
\hline $\begin{array}{l}\text { Age at surgery, year, mean } \\
\text { (standard error: SE) }\end{array}$ & $71.6 \pm 0.19$ & $72.1 \pm 0.27$ & 0.125 \\
\hline Gender & & & 0.420 \\
\hline Male & $1942(57.8)$ & $848(59.1)$ & \\
\hline Female & $1418(42.2)$ & $588(40.9)$ & \\
\hline Race & & & 0.751 \\
\hline Caucasian & $2973(88.5)$ & $1266(88.2)$ & \\
\hline Other & $387(11.5)$ & $170(11.8)$ & \\
\hline Tumor location & & & 0.698 \\
\hline Pelvic tumors & $2248(66.9)$ & $969(67.5)$ & \\
\hline Ureteral and/or multifocal tumors & $1112(33.1)$ & $467(32.5)$ & \\
\hline T stage & & & 0.997 \\
\hline $\mathrm{T} 1$ & $1083(32.2)$ & $458(31.9)$ & \\
\hline $\mathrm{T} 2$ & $663(19.7)$ & $284(19.8)$ & \\
\hline $\mathrm{T} 3$ & $1403(41.8)$ & $603(42.0)$ & \\
\hline $\mathrm{T} 4$ & $211(6.3)$ & $91(6.3)$ & \\
\hline N stage & & & 0.297 \\
\hline No & $3053(90.9)$ & $1312(91.4)$ & \\
\hline N1 & $182(5.4)$ & $76(5.3)$ & \\
\hline $\mathrm{N} 2$ & $125(3.7)$ & $68(4.7)$ & \\
\hline M stage & & & 0.179 \\
\hline M0 & $3263(97.1)$ & $1384(96.4)$ & \\
\hline M1 & $97(2.9)$ & $52(3.6)$ & \\
\hline Grade & & & 0.374 \\
\hline G1-2 & $627(18.7)$ & $289(20.1)$ & \\
\hline G3-4 & $2733(81.3)$ & $1174(81.8)$ & \\
\hline AJCC-TNM & & & 0.974 \\
\hline I & 1058 (31.5) & $446(31.1)$ & \\
\hline II & 625 (18.6) & $263(18.3)$ & \\
\hline III & $1190(35.4)$ & $514(35.8)$ & \\
\hline IV & 487 (14.5) & $213(14.8)$ & \\
\hline
\end{tabular}

At 5 years after RNU, 19.8\% (946/4769) of the UTUC patients died. According to the most recent 
pathological prognostic group classification, the 3 -year CSS rates of patients with stage I, II, III and IV disease were $93.5 \%, 86.7 \%, 75.4 \%$ and $49.0 \%$, and the 5 -year CSS rates of patients with stage I, II, III and IV disease were $89.7 \%, 81.1 \%, 66.1 \%$ and $38.1 \%$, respectively $(P<0.001$, Figure 1). The stratified Kaplan-Meier plots shown in Table 2 illustrate the effects of various factors on the actual CSS rates. In the training cohort, the univariable analyses revealed that age, gender, the tumor stage, the node stage, the metastasis stage and the grade were statistically significantly associated with CSS (all $P<0.001$ ).

The multivariate Cox regression model identified age, the tumor stage, the node stage, the metastasis stage and the grade as covariates associated with survival (all $P<0.001$ ) (Table 3). The nomogram subsequently generated based on this multivariate analysis is shown in Figure 2.

In the training cohort, the nomogram exhibited discrimination power superior to that of the 8th AJCC
TNM classification (Harrell's concordance index [C-index], 0.74 vs. $0.71 ; P<0.001)$. The bootstrapcorrected C-index of the nomogram was 0.74 , which was inferior to that of the 8th AJCC TNM staging system (C-index, 0.70; $P<0.001)$. The receiver operating curve is illustrated in Figure 3. The calibration plots and decision curve analysis suggested that the accuracy in predicting CSS was good as shown in Figure 4, Figure 5 and Table 4.

\section{Discussion}

CSS after RNU for UTUC may be highly variable [2]. Clinicopathological factors, such as the T stage, $\mathrm{N}$ stage and G stage, represent established predictors of prognosis [5, 14]. Few nomograms incorporate various variables as predictors of prognosis [9-11]. However, a unified standard for nomograms is lacking.
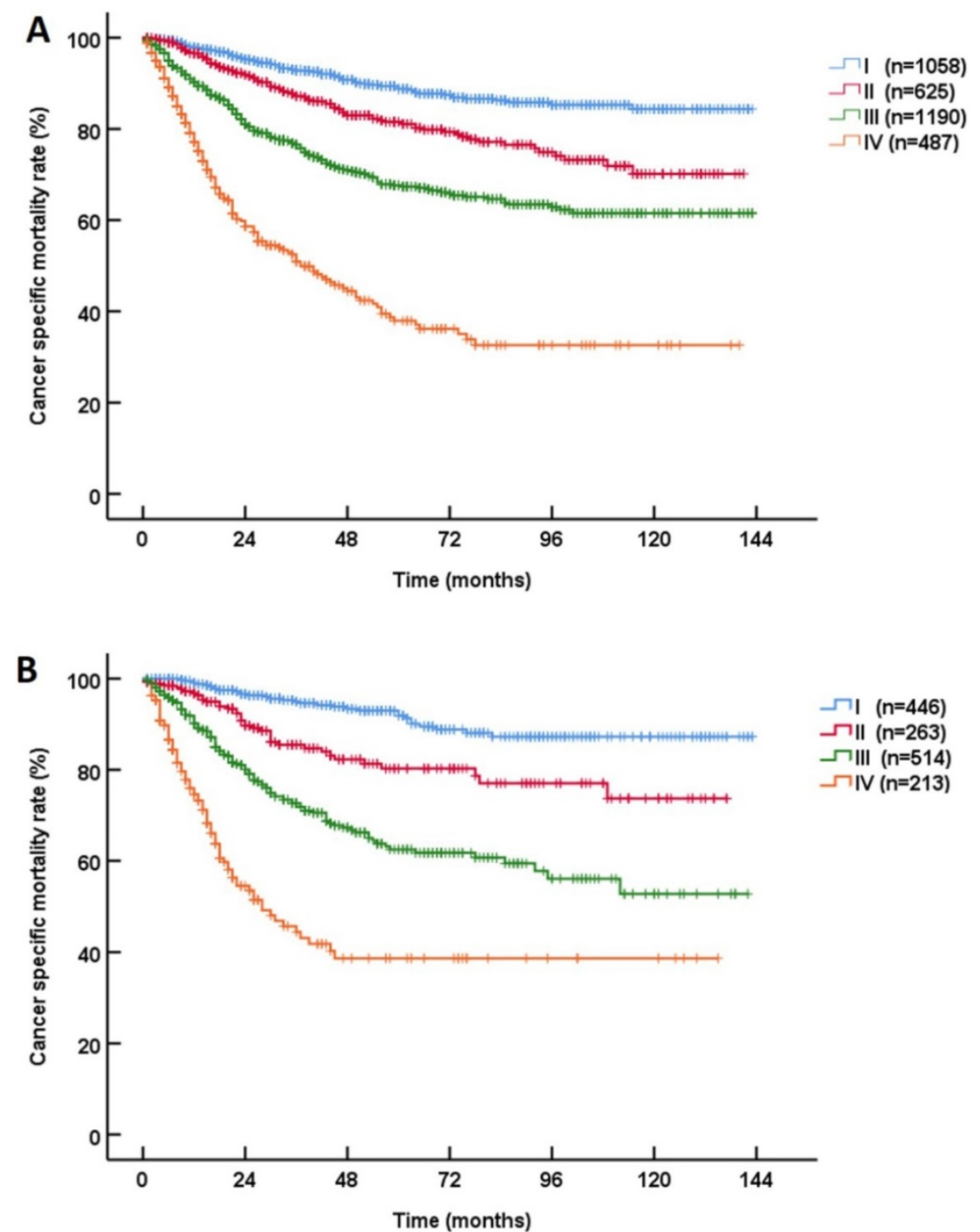

Figure 1. Kaplan-Meier Cancer-specific survival (CSS) curves of patients with invasive upper tract urothelial carcinoma in different AJCC-pathological prognostic group classifications. A: training cohort; B: validation cohort. 


$\begin{array}{llllllllllll} & 0 & 10 & 20 & 30 & 40 & 50 & 60 & 70 & 80 & 90 & 100\end{array}$

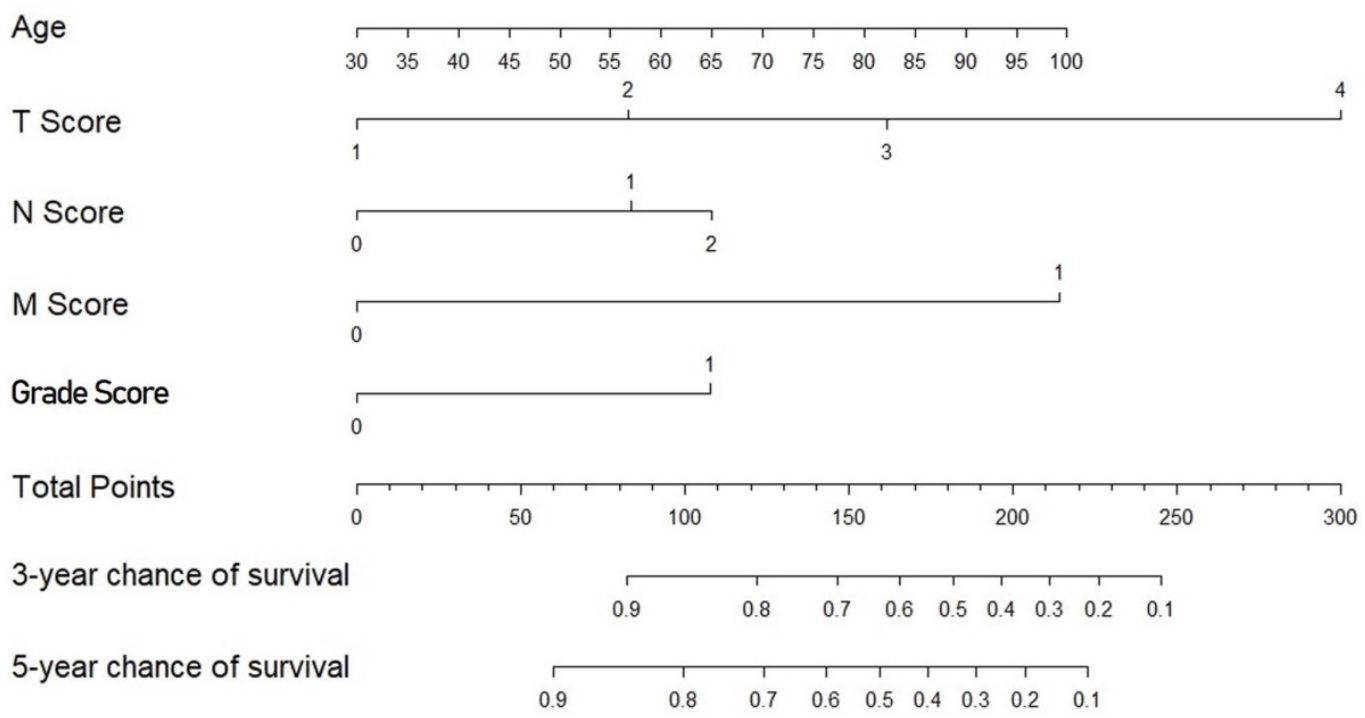

Figure 2. Nomogram predicting survival in patients with invasive upper tract urothelial carcinoma.

Table 2. Cancer specific mortality in patients with UTUC

\begin{tabular}{|c|c|c|c|c|c|c|}
\hline \multirow[t]{2}{*}{ Variable } & \multicolumn{3}{|l|}{ Training set } & \multicolumn{3}{|l|}{ Validation set } \\
\hline & 3-year CSS & 5-year CSS & $P$ & 3-year CSS & 5-year CSS & $P$ \\
\hline Age at surgery (year) & & & $<0.001$ & & & 0.002 \\
\hline$\leq 73$ & $84.5(82.5-86.5)$ & $78.2(75.8-80.6)$ & & $81.3(78.2-84.4)$ & $76.1(72.4-79.8)$ & \\
\hline$>73$ & $77.2(74.8-79.6)$ & $68.9(66.0-71.8)$ & & $77.1(73.4-80.8)$ & $70.1(65.6-74.6)$ & \\
\hline Gender & & & 0.002 & & & 0.025 \\
\hline Male & $83.2(81.2-85.2)$ & $76.8(74.4-79.2)$ & & $80.5(77.4-83.6)$ & $75.0(71.3-78.7)$ & \\
\hline Female & $78.0(75.5-80.5)$ & $70.4(67.5-73.3)$ & & $77.2(73.3-81.1)$ & $70.8(66.1-75.5)$ & \\
\hline Race & & & 0.299 & & & 0.341 \\
\hline Caucasian & $81.4(79.8-83.0)$ & $74.6(72.6-76.6)$ & & $79.4(76.9-81.9)$ & $73.7(70.6-76.8)$ & \\
\hline Other & $77.5(72.8-82.2)$ & $70.0(64.3-75.7)$ & & $77.4(70.1-84.7)$ & $69.9(60.9-78.9)$ & \\
\hline Tumor location & & & 0.975 & & & 0.693 \\
\hline Pelvic tumors & $80.8(78.8-82.8)$ & $73.7(71.3-76.1)$ & & $79.2(76.3-82.1)$ & $73.4(69.9-76.9)$ & \\
\hline Ureteral and/or multifocal tumors & $81.3(78.6-84.0)$ & $74.8(71.5-78.1)$ & & $79.1(74.6-83.6)$ & $72.9(67.6-78.2)$ & \\
\hline T stage & & & $<0.001$ & & & $<0.001$ \\
\hline $\mathrm{T} 1$ & $92.3(90.5-94.1)$ & $88.2(85.8-90.6)$ & & $94.1(91.7-96.5)$ & $91.1(87.8-94.4)$ & \\
\hline $\mathrm{T} 2$ & $85.9(82.8-89.0)$ & $79.8(75.9-83.7)$ & & $84.4(79.3-89.5)$ & $78.8(72.7-84.9)$ & \\
\hline $\mathrm{T} 3$ & $73.8(71.1-76.5)$ & $64.5(61.2-67.8)$ & & $68.5(64.0-73.0)$ & $58.3(52.8-63.8)$ & \\
\hline $\mathrm{T} 4$ & $45.2(36.8-53.6)$ & $30.4(21.4-39.4)$ & & $39.8(26.1-53.5)$ & $39.8(26.1-53.5)$ & \\
\hline N stage & & & $<0.001$ & & & $<0.001$ \\
\hline No & $83.3(81.5-85.1)$ & $76.6(74.6-78.6)$ & & $81.8(79.4-84.2)$ & $76.1(73.2-79.0)$ & \\
\hline N1 & $53.8(44.6-63.0)$ & $43.3(33.1-53.5)$ & & $50.3(35.6-65.0)$ & $37.3(21.8-52.8)$ & \\
\hline N2 & $51.5(39.5-63.5)$ & $38.1(21.6-54.6)$ & & $38.8(19.4-58.2)$ & $38.8(19.4-58.2)$ & \\
\hline M stage & & & $<0.001$ & & & $<0.001$ \\
\hline M0 & $82.2(80.6-83.8)$ & $75.2(73.2-77.2)$ & & $81.0(78.6-83.4)$ & $74.9(72.0-77.8)$ & \\
\hline M1 & $24.5(10.0-39.0)$ & $16.3(0-32.6)$ & & $19.7(5.0-34.4)$ & $19.7(5.0-34.4)$ & \\
\hline Grade & & & $<0.001$ & & & $<0.001$ \\
\hline G1-2 & $93.5(91.3-95.7)$ & $90.5(87.8-93.2)$ & & $94.7(91.8-97.6)$ & $93.5(90.2-96.8)$ & \\
\hline G3-4 & 77.7 (75.9-79.5) & $69.6(67.4-71.8)$ & & 74.5 (71.6-77.4) & $66.7(63.0-70.4)$ & \\
\hline AJCC-TNM & & & $<0.001$ & & & $<0.001$ \\
\hline I & $92.9(91.1-94.7)$ & 88.7 (86.3-91.1) & & $94.9(92.5-97.3)$ & $91.8(88.7-94.9)$ & \\
\hline II & $87.2(84.3-90.1)$ & $81.5(77.8-85.2)$ & & $85.5(80.6-90.4)$ & $80.3(74.0-86.6)$ & \\
\hline III & $76.6(73.9-79.3)$ & $67.6(64.1-71.1)$ & & $72.6(67.9-77.3)$ & $62.5(56.6-68.4)$ & \\
\hline IV & $50.9(45.2-56.6)$ & $37.9(31.2-44.6)$ & & $44.5(35.5-53.5)$ & $38.7(29.3-48.1)$ & \\
\hline
\end{tabular}

Using the SEER database, we developed a concise nomogram for the prediction of CSS in patients with UTUC and verified its clinical application value. The nomogram, which includes only 5 of the most common factors, is concise and has high applicability. The results were obtained in the training cohort and tested in the validation cohort. This model represents an individualized prognostic 
tool for patients treated with RNU based on the largest UTUC data set.

Invasive UTUC has a very poor prognosis [1, 2]. In this study, RNU afforded a 3-year and 5-year CSS rate of $69 \%$ and $73 \%$, respectively [6]. Several single-center series involving $>200$ patients published to date have demonstrated 5-year CSS rates ranging from $61 \%-76 \%$ after RNU $[16,17]$. According to the currently accepted definition, the AJCC TNM staging system is considered among the most important prediction tools for UTUC. In our study, the 5-year CSS rates of the patients with stage I, II, III and IV disease were $87.3 \%, 79.3 \%, 64.9 \%$ and $32.8 \%$, respectively. Although this finding represents a monumental step toward the goal of precision medicine, this work was published only very recently.
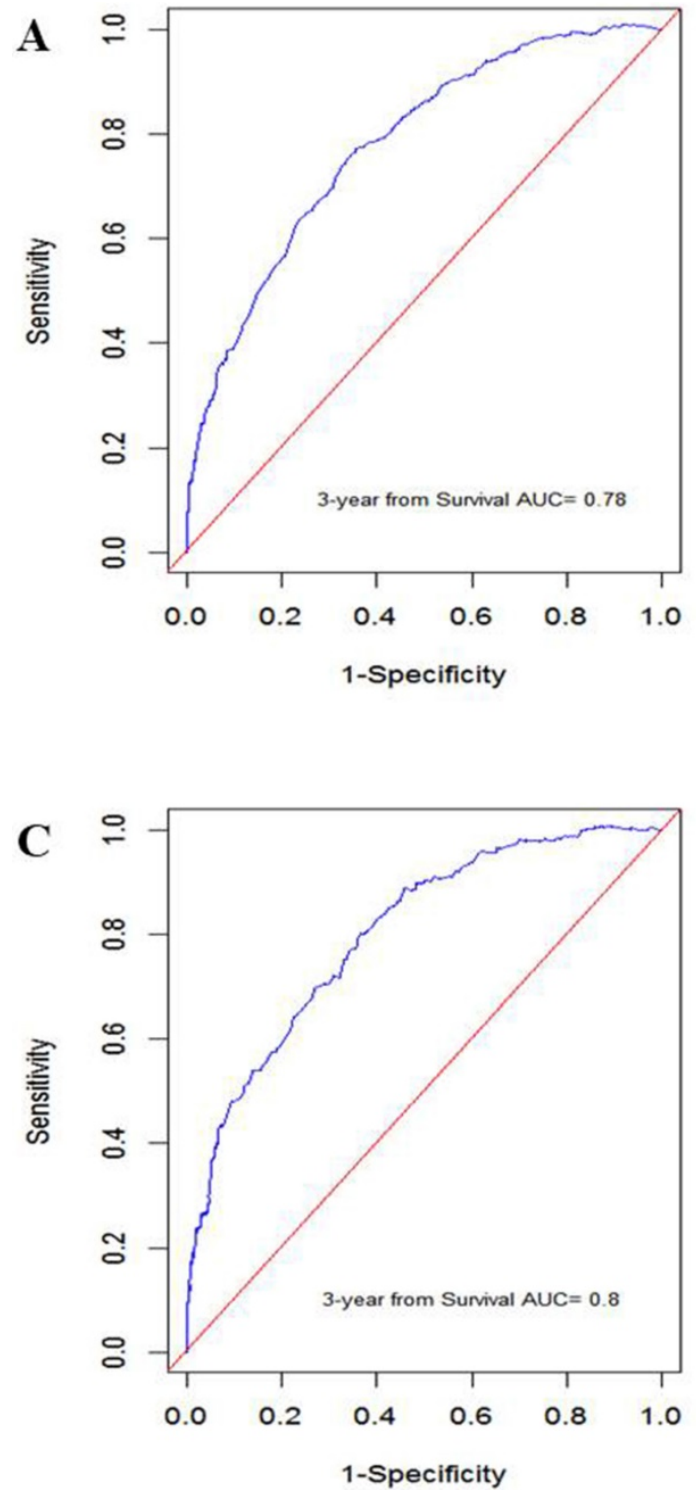

Table 3. Multivariate analysis of the clinicopathological factors used to predict the cancer specific mortality of patients with UTUC

\begin{tabular}{|c|c|c|c|c|c|c|}
\hline \multirow[t]{2}{*}{ Variable } & \multicolumn{3}{|c|}{ Training set } & \multicolumn{3}{|c|}{ Validation set } \\
\hline & HR & $95 \% \mathrm{CI}$ & $P$ & HR & $95 \% \mathrm{CI}$ & $P$ \\
\hline Age at surgery & 1.748 & $1.50-2.04$ & $<0.001$ & 1.030 & $1.02-1.04$ & $<0.001$ \\
\hline T stage & & & $<0.001$ & & & $<0.001$ \\
\hline T1 & Ref & Ref & Ref & Ref & Ref & Ref \\
\hline $\mathrm{T} 2$ & 1.750 & $1.33-2.30$ & $<0.001$ & 1.580 & $1.00-2.48$ & 0.049 \\
\hline T3 & 2.888 & $2.30-3.52$ & $<0.001$ & 3.670 & $2.53-5.33$ & $<0.001$ \\
\hline $\mathrm{T} 4$ & 5.753 & $4.27-7.75$ & $<0.001$ & 6.720 & $4.05-11.16$ & $<0.001$ \\
\hline $\mathrm{N}$ stage & & & $<0.001$ & & & 0.001 \\
\hline No & Ref & Ref & Ref & Ref & Ref & Ref \\
\hline N1 & 1.511 & $1.16-2.00$ & 0.003 & 1.830 & $1.23-2.72$ & 0.003 \\
\hline N2 & 1.696 & $1.23-2.34$ & 0.001 & 2.030 & $1.23-3.36$ & 0.006 \\
\hline M stage & & & & & & $<0.001$ \\
\hline M0 vs. M1 & 4.229 & $3.05-5.86$ & $<0.001$ & 3.371 & $2.20-5.17$ & \\
\hline Grade & & & & & & $<0.001$ \\
\hline G1-2 vs. G3-4 & 1.207 & $1.50-2.04$ & 0.001 & 1.400 & $1.18-1.66$ & \\
\hline
\end{tabular}
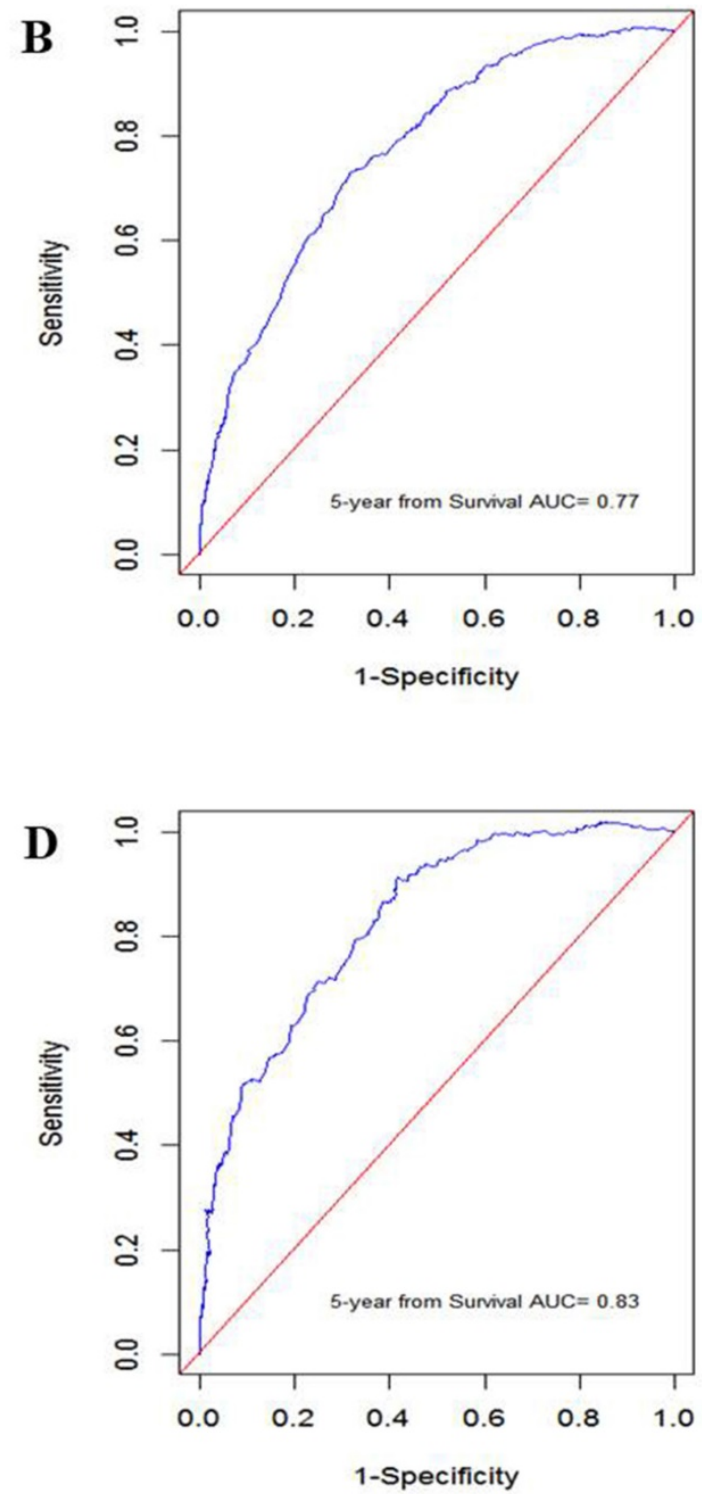

Figure 3. ROC curve generated by the nomogram. A: 3-year CSS in the training cohort. B: 5-year CSS in the training cohort. C: 3-year CSS in the validation cohort. D: 5-year CSS in the validation cohort. 
A

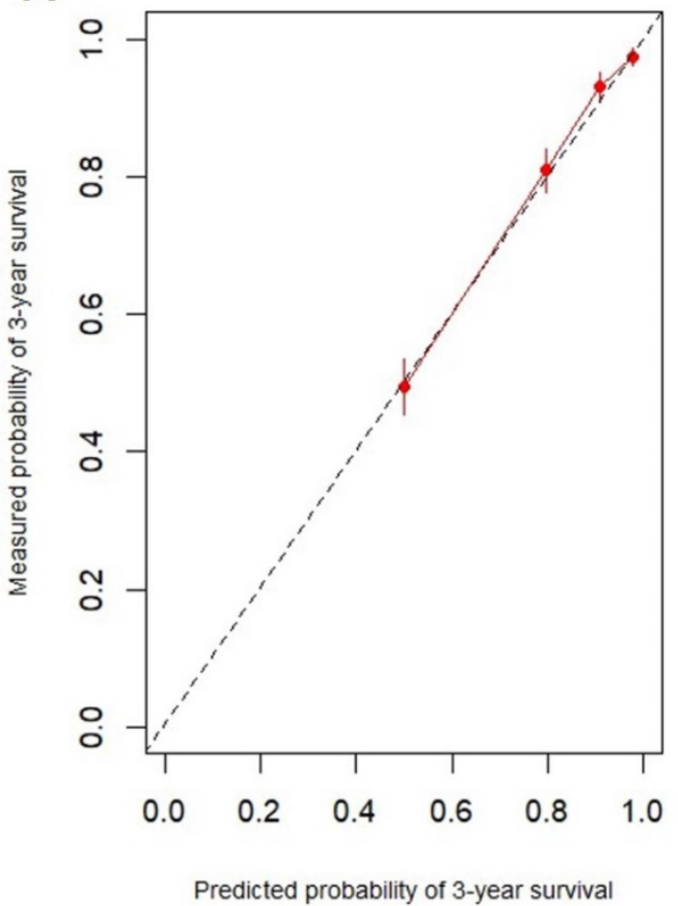

C

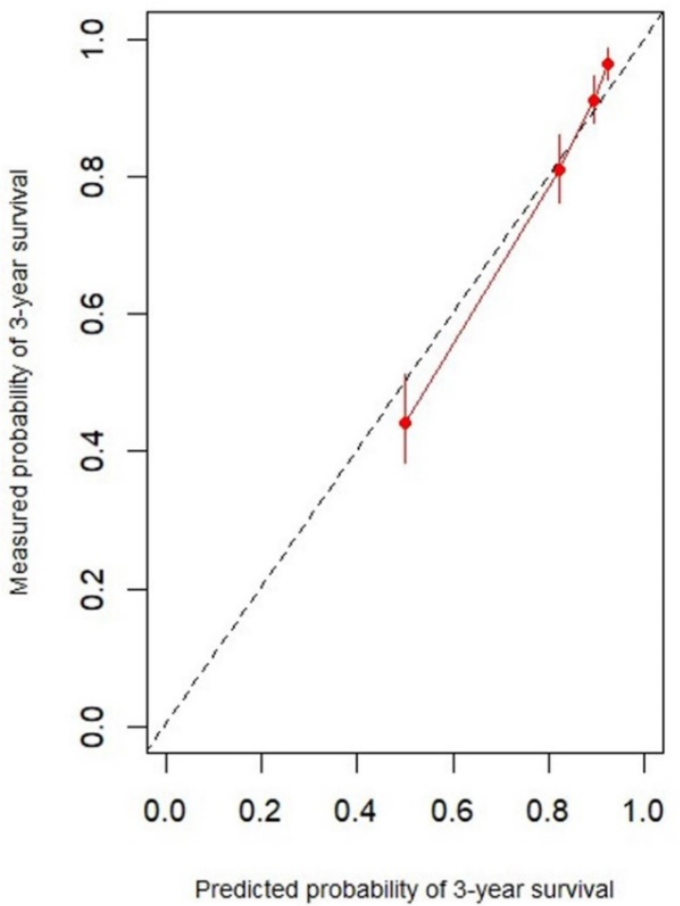

B

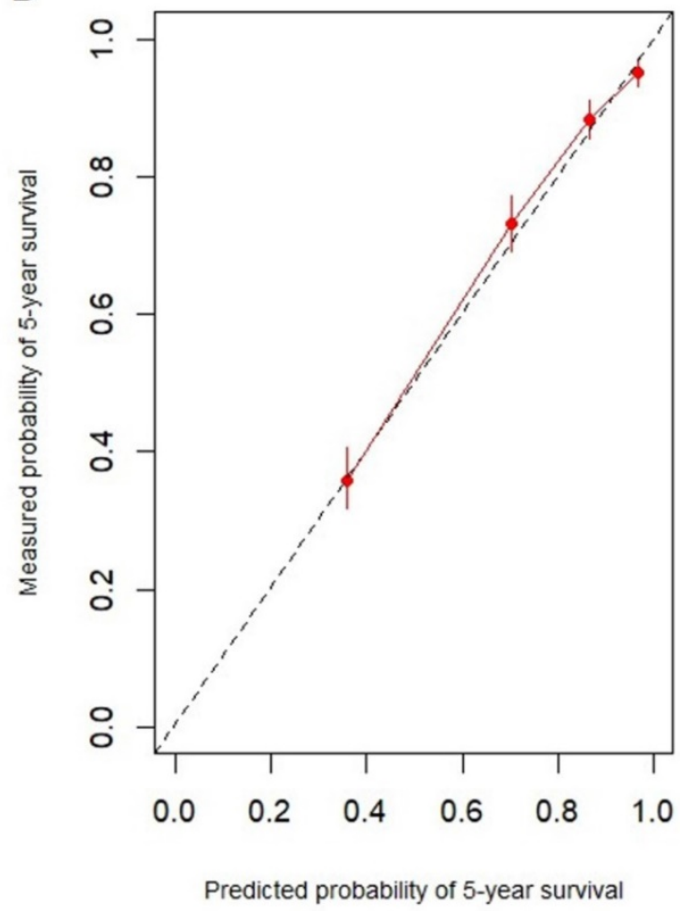

D

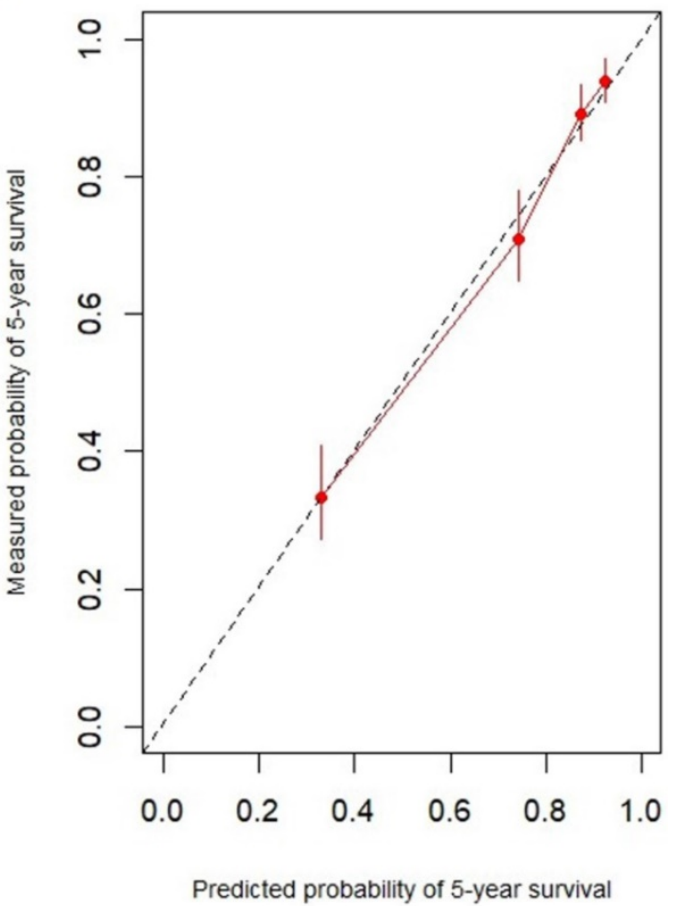

Figure 4. The prognostic accuracy of the nomogram in patients with invasive upper tract urothelial carcinoma. A: 3-year CSS in the training cohort. B: 5-year CSS in the training cohort. C: 3-year CSS in the validation cohort. D: 5-year CSS in the validation cohort.

The main prognostic factors include preoperative and postoperative factors. Age, the tumor stage, the node stage, the metastasis stage and the grade, which are the most common factors, were included in this nomogram. The above factors have been shown to be independent prognostic predictors.
This nomogram achieved a greater accuracy of CSS than the AJCC-TNM classification. The age at the time of RNU is an independent indicator of patient outcomes [18]. The primary recognized prognostic factors are the tumor stage and grade [18-23]. 

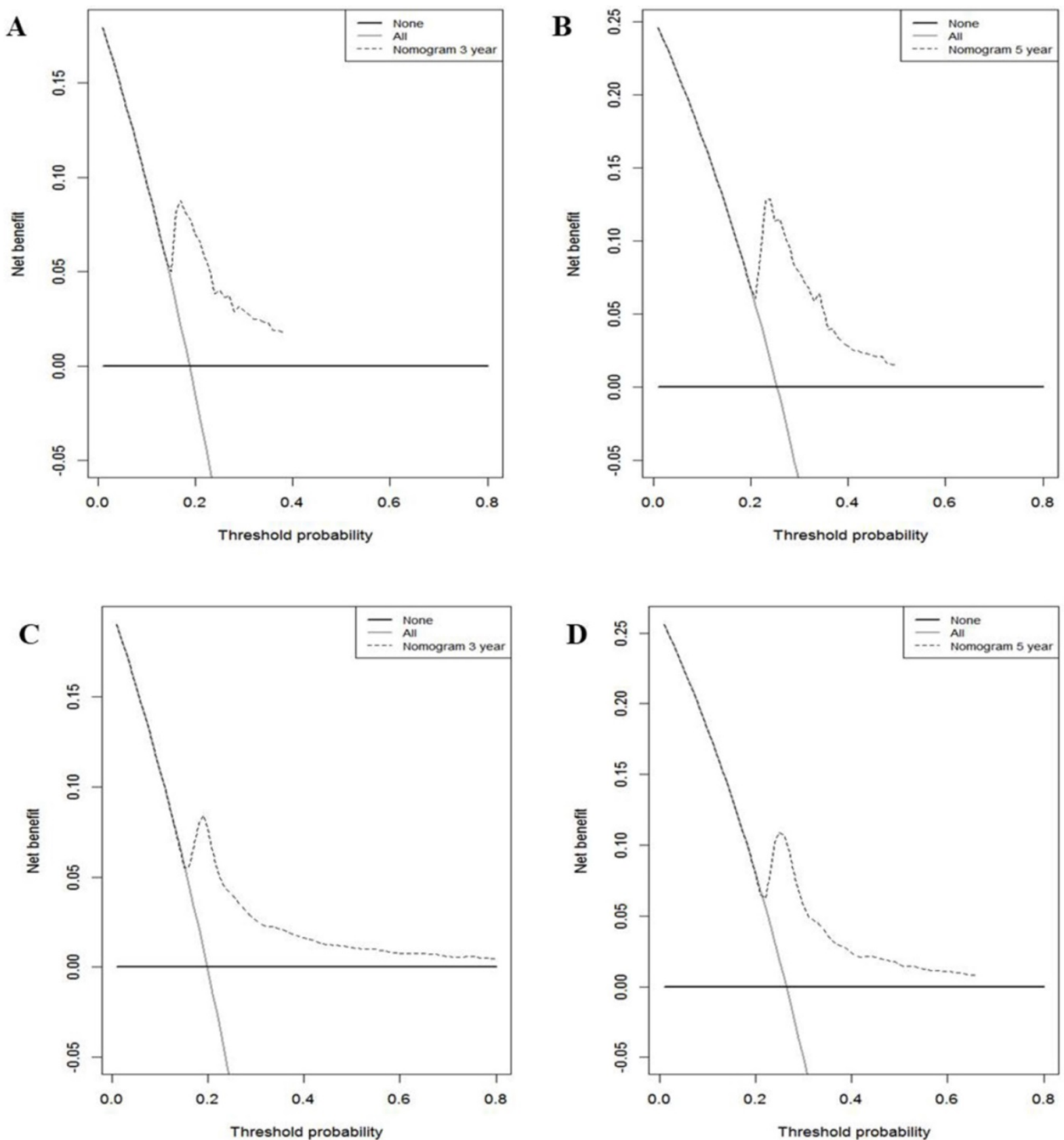

Figure 5. Decision curve analysis used to assess the clinical usefulness of the nomogram. A: 3-year CSS in the training cohort. B: 5-year CSS in the training cohort. C: 3-year CSS in the validation cohort. D: 5-year CSS in the validation cohort.

Table 4. Predictive accuracy of the staging system

\begin{tabular}{llll}
\hline Stage & C-index (Training set) & $\begin{array}{l}\text { Bootstrap C-index } \\
\text { (Training set) }\end{array}$ & $\begin{array}{l}\text { C-index } \\
\text { (Validation set) }\end{array}$ \\
\hline AJCC-TNM & 0.71 & 0.70 & 0.73 \\
Nomogram & 0.74 & 0.75 & 0.77 \\
\hline
\end{tabular}

Different nomograms based on the French Collaborative National Database that incorporate several variables have been developed [9-11]. In 2012, Eugene K. Cha et al. developed a seven-factor prognostic model for the prediction of CSS after RNU for UTUC [6]. The model predicted $26.5 \%$ of noninvasive urothelial carcinoma patients. In this study, only age, the $\mathrm{T}$ classification, the lymph node status, lymphovascular invasion (LVI), and the tumor architecture were independent predictors of CSS (all $P$ values $<0.005$ ). However, the grade and concomitant carcinoma in situ (CIS) were still incorporated into this model. It could be more helpful to establish a uniform model for daily urological practice than choose among models. In the future, statistical prediction models of this cancer could be evaluated, and those that meet all AJCC criteria should be endorsed.

There are some several limitations as follows: 1 ) limitations inherent to retrospective analyses; 2 ) some 
information was not included in this study; according to previous studies, neoadjuvant chemotherapy and adjuvant chemotherapy significantly benefit overall survival, but the current nomogram was not compared with well-developed models; and 3) we failed to present the difference among established nomograms. However, the clinicopathological features used to establish the nomogram have been used in previous studies. The nomograms did not incorporate the same variables, or have difference prognosis outcomes. Our nomogram only includes 5 of the most common factors. Because some factors could not be collected, such comparisons could not be performed. In addition, we compared the newly developed nomogram with the 8th AJCC TNM classification, which is considered among the most important prediction tools for UTUC. The medical records of the patients included in this study were randomly (7:3) divided into the training and validation cohorts, and number of patients per cohort slightly differed from that in previous studies. Nevertheless, we obtained the same results. Although internal and external validation of the data set was performed, the results still need to be externally verified in large samples. However, we believe that different established nomograms could be verified with unified data.

\section{Conclusion}

A concise novel nomogram that is superior to the current AJCC TNM staging system was established for the prediction of CSS after RNU for invasive UTUC. This model can be an important tool that aids clinical decision making. The clinical value of this tool should be validated in prospective, multi-institutional studies.

\section{Abbreviations}

AJCC/UICC: American Joint Committee on Cancer/Union Internationale Contre le Cancer; AUC: receiver-operating characteristic curve; CSS: cancerspecific survival; EAU: European Association of Urology; NCCN: National Comprehensive Cancer Network; RNU: radical nephroureterectomy; SEER: Surveillance, Epidemiology and End Results; TNM: tumor-node-metastasis; UC: urothelial carcinomas; UTUC: upper tract urothelial carcinoma.

\section{Acknowledgements}

We are grateful to the authors and the enrolled patients, their families, and the referring physicians. We also gratefully acknowledge the contribution of all colleagues in this study. Additionally, we thank our families for their support.

\section{Funding}

This work was supported by the National Natural Science Foundation of China (Grant No. 81902610), Science and Technology Planning Project of Guangdong Province of China (Grant No. B2020197).

\section{Availability of data and materials}

The datasets used and/or analyzed during the present study are available from the corresponding author upon reasonable request.

\section{Author contributions}

KF Xiao and ZQ Liu had full access to all data in the study and take responsibility for the integrity of the data and the accuracy of the data analysis. Study conception and design: ZS Li, XY Li, YH Li, KF Xiao and ZQ Liu. Acquisition of the data: All authors. Analysis and interpretation of the data: All authors. Drafting of the manuscript: ZS Li, XY Li and YH Li. Critical revision of the manuscript for important intellectual content: KF Xiao and ZQ Liu. Statistical analysis: All authors. Obtaining funding: ZS Li. Administrative, technical, and material support: KF Xiao and ZQ Liu. Supervision: ZS Li, XY Li, KF Xiao and ZQ Liu. Other (specify): None.

\section{Ethics approval and consent to participate}

The present study was approved by the Ethics Committee of Shenzhen People's Hospital (LL-KY-2020125).

\section{Competing Interests}

The authors have declared that no competing interest exists.

\section{References}

1. Roupret M, Babjuk M, Comperat E, Zigeuner R, Sylvester RJ, Burger M, et al. European Association of Urology Guidelines on Upper Urinary Tract Urothelial Cell Carcinoma: 2015 Update. Eur Urol. 2015; 68: 868-79.

2. Roupret M, Babjuk M, Comperat E, Zigeuner R, Sylvester RJ, Burger M, et al. European Association of Urology Guidelines on Upper Urinary Tract Urothelial Carcinoma: 2017 Update. Eur Urol. 2018; 73: 111-22.

3. Krabbe LM, Westerman ME, Bagrodia A, Gayed BA, Khalil D, Kapur P, et al. Surgical management of the distal ureter during radical nephroureterectomy is an independent predictor of oncological outcomes: results of a current series and a review of the literature. Urol Oncol. 2014; 32: 54 e19-26.

4. Margulis V, Shariat SF, Matin SF, Kamat AM, Zigeuner R, Kikuchi E, et al. Outcomes of radical nephroureterectomy: a series from the Upper Tract Urothelial Carcinoma Collaboration. Cancer. 2009; 115: 1224-33.

5. Clements T, Messer JC, Terrell JD, Herman MP, Ng CK, Scherr DS, et al. High-grade ureteroscopic biopsy is associated with advanced pathology of upper-tract urothelial carcinoma tumors at definitive surgical resection. J Endourol. 2012; 26: 398-402.

6. Lughezzani G, Burger M, Margulis V, Matin SF, Novara G, Roupret M, et al. Prognostic factors in upper urinary tract urothelial carcinomas: a comprehensive review of the current literature. Eur Urol. 2012; 62: 100-14.

7. Simsir A, Sarsik B, Cureklibatir I, Sen S, Gunaydin G, Cal C. Prognostic factors for upper urinary tract urothelial carcinomas: stage, grade, and smoking status. Int Urol Nephrol. 2011; 43: 1039-45.

8. Roupret M, Babjuk M, Burger M, Capoun O, Cohen D, Comperat EM, et al. European Association of Urology Guidelines on Upper Urinary Tract Urothelial Carcinoma: 2020 Update. Eur Urol. 2020;20:30427-9

9. Roupret M, Hupertan V, Seisen T, Colin P, Xylinas E, Yates DR, et al. Prediction of cancer specific survival after radical nephroureterectomy for 
upper tract urothelial carcinoma: development of an optimized postoperative nomogram using decision curve analysis. J Urol. 2013; 189: 1662-9.

10. Seisen T, Colin P, Hupertan V, Yates DR, Xylinas E, Nison L, et al. Postoperative nomogram to predict cancer-specific survival after radical nephroureterectomy in patients with localised and/or locally advanced upper tract urothelial carcinoma without metastasis. BJU Int. 2014; 114: 733-40.

11. Yates DR, Hupertan V, Colin P, Ouzzane A, Descazeaud A, Long JA, et al. Cancer-specific survival after radical nephroureterectomy for upper urinary tract urothelial carcinoma: proposal and multi-institutional validation of a post-operative nomogram. British journal of cancer. 2012; 106: 1083-8.

12. Fang Y, Cen JJ, Cao JZ, Huang Y, Feng ZH, Lu J, et al. Overexpression of EIF5A2 in upper urinary tract urothelial carcinoma is a new independent prognostic marker of survival. Future Oncol. 2019; 15: 2009-18.

13. Yoshida $\mathrm{T}$, Kobayashi $\mathrm{T}$, Kawaura $\mathrm{T}$, Miyake $\mathrm{M}$, Ito $\mathrm{K}$, Okuno $\mathrm{H}$, et al. Development and external validation of a preoperative nomogram for predicting pathological locally advanced disease of clinically localized upper urinary tract carcinoma. Cancer Med. 2020; 9: 3733-41.

14. Rink M, Robinson BD, Green DA, Cha EK, Hansen J, Comploj E, et al. Impact of histological variants on clinical outcomes of patients with upper urinary tract urothelial carcinoma. J Urol. 2012; 188: 398-404.

15. Brierley. JD, Gospodarowicz MK, C. W. Comments on the TNM classification of malignant tumours--8th edition. Oxford, UK: John Wiley \& Sons Publisher. 2017.

16. Hall MC, Womack S, Sagalowsky AI, Carmody T, Erickstad MD, Roehrborn CG. Prognostic factors, recurrence, and survival in transitional cell carcinoma of the upper urinary tract: a 30-year experience in 252 patients. Urology. 1998; 52: 594-601.

17. Secin FP, Koppie TM, Salamanca JI, Bokhari S, Raj GV, Olgac S, et al Evaluation of regional lymph node dissection in patients with upper urinary tract urothelial cancer. International journal of urology : official journal of the Japanese Urological Association. 2007; 14: 26-32.

18. Park J, Habuchi T, Arai Y, Ohyama C, Inoue T, Hatakeyama S, et al. Reassessment of prognostic heterogeneity of $\mathrm{pT} 3$ renal pelvic urothelial carcinoma: analysis in terms of proposed pT3 subclassification systems. J Urol. 2014; 192: 1064-71.

19. Chromecki TF, Bensalah K, Remzi M, Verhoest G, Cha EK, Scherr DS, et al. Prognostic factors for upper urinary tract urothelial carcinoma. Nat Rev Urol. 2011; 8: 440-7.

20. Kim SH, Song MK, Joung JY, Chung J, Lee KH, Seo HK. Significant clinicopathologic prognostic factors for bladder recurrence, progression, and cancer-specific survival after surgery among patients with upper urinary tract urothelial carcinoma. Investig Clin Urol. 2019; 60: 432-42.

21. Kuroda K, Asakuma J, Horiguchi A, Tasaki S, Yoshii H, Sato A, et al. Prognostic factors for upper urinary tract urothelial carcinoma after nephroureterectomy. Urol Int. 2012; 88: 225-31.

22. Liu Y, Lu J, Hong K, Huang Y, Ma L. Independent prognostic factors for initial intravesical recurrence after laparoscopic nephroureterectomy for upper urinary tract urothelial carcinoma. Urol Oncol. 2014; 32: 146-52.

23. Morizane $S$, Iwamoto $H$, Masago $T$, Yao A, Isoyama $T$, Sejima $T$, et al. Preoperative prognostic factors after radical nephroureterectomy in patients with upper urinary tract urothelial carcinoma. Int Urol Nephrol. 2013; 45: 99-106. 